\title{
Supplementary material: SoilGrids 2.0: producing quality-assessed soil information for the globe
}

Luis M. de Sousa ${ }^{1}$, Laura Poggio ${ }^{1}$, Niels H. Batjes ${ }^{1}$, Gerard B. M. Heuvelink ${ }^{1}$, Bas Kempen ${ }^{1}$, Eloi Ribeiro ${ }^{1}$, and David Rossiter ${ }^{1}$

${ }^{1}$ ISRIC - World Soil Information - Wageningen (NL)

\section{Geographic density of observations}

The number of locations with at least one observation for each of the soil properties were summed in each cell of an ISEAG grid of resolution 6 and aperture 3 (see section on the stratification methodology in the main paper for further details).
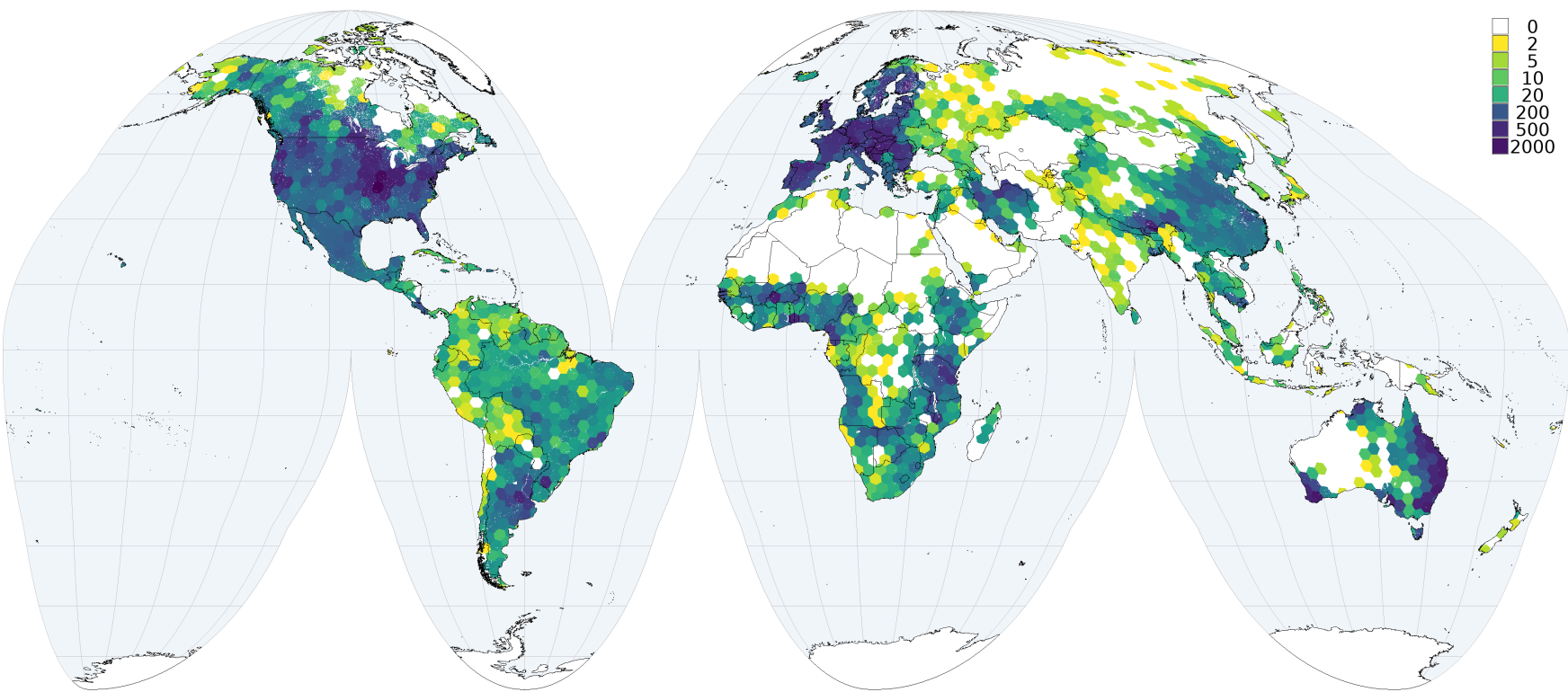

Figure S1. Number of observations for soil organic carbon. 


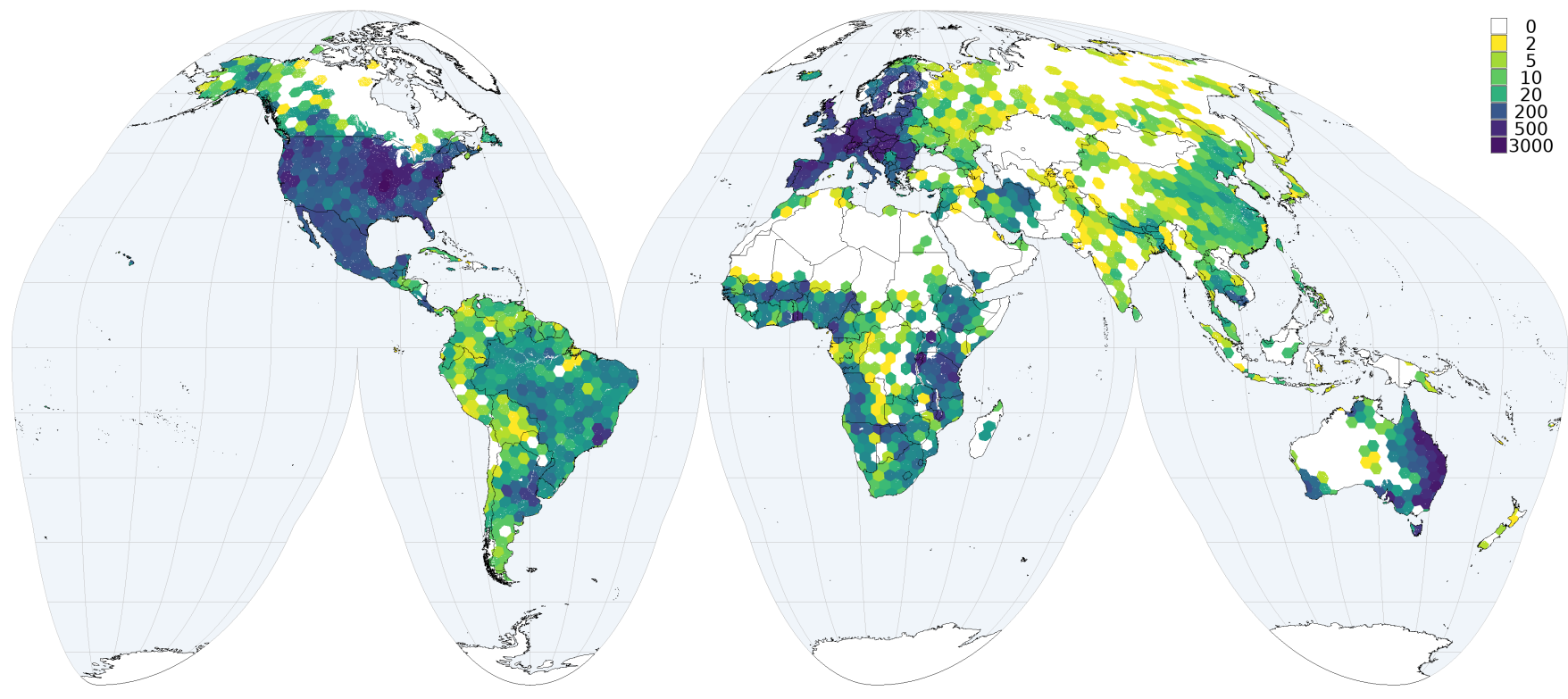

Figure S2. Number of observations for soil $\mathrm{pH}_{\text {water }}$.
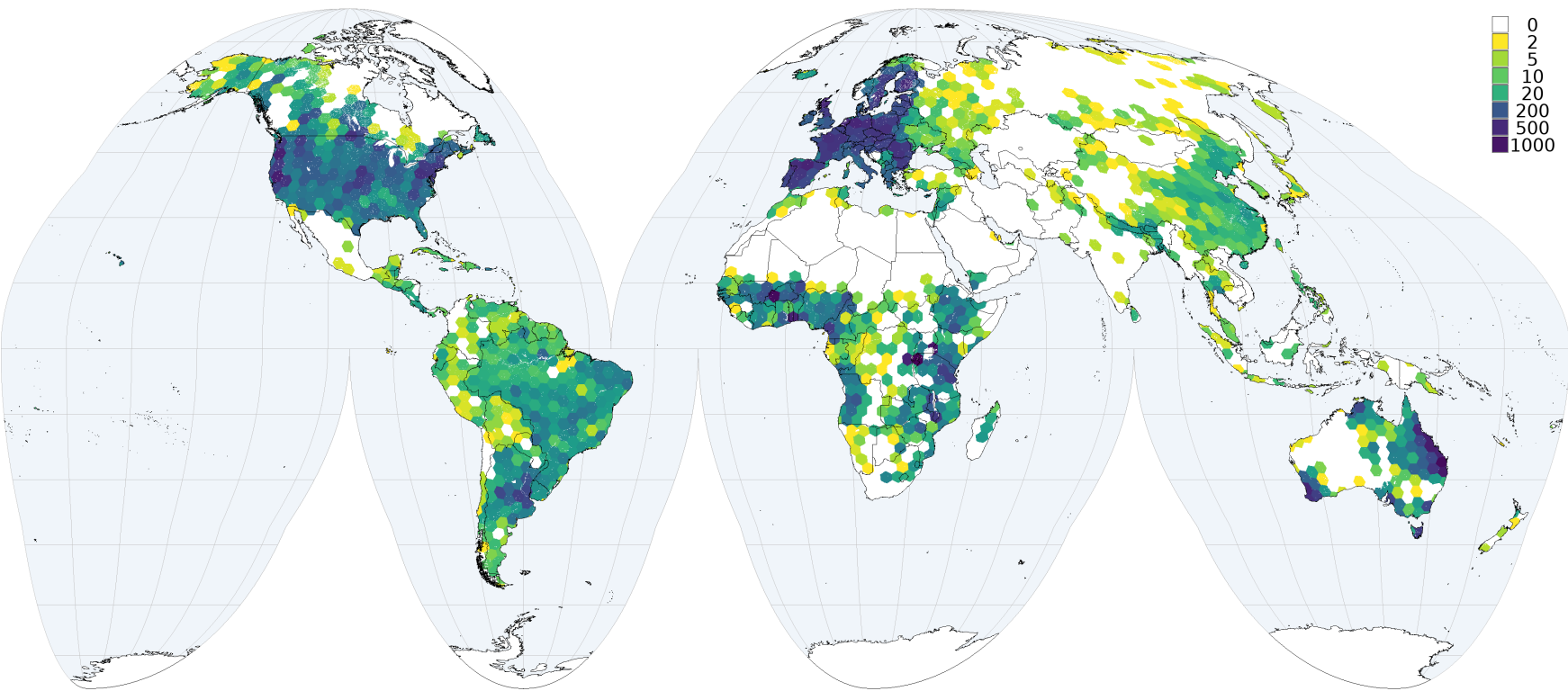

Figure S3. Number of observations for soil nitrogen content. 


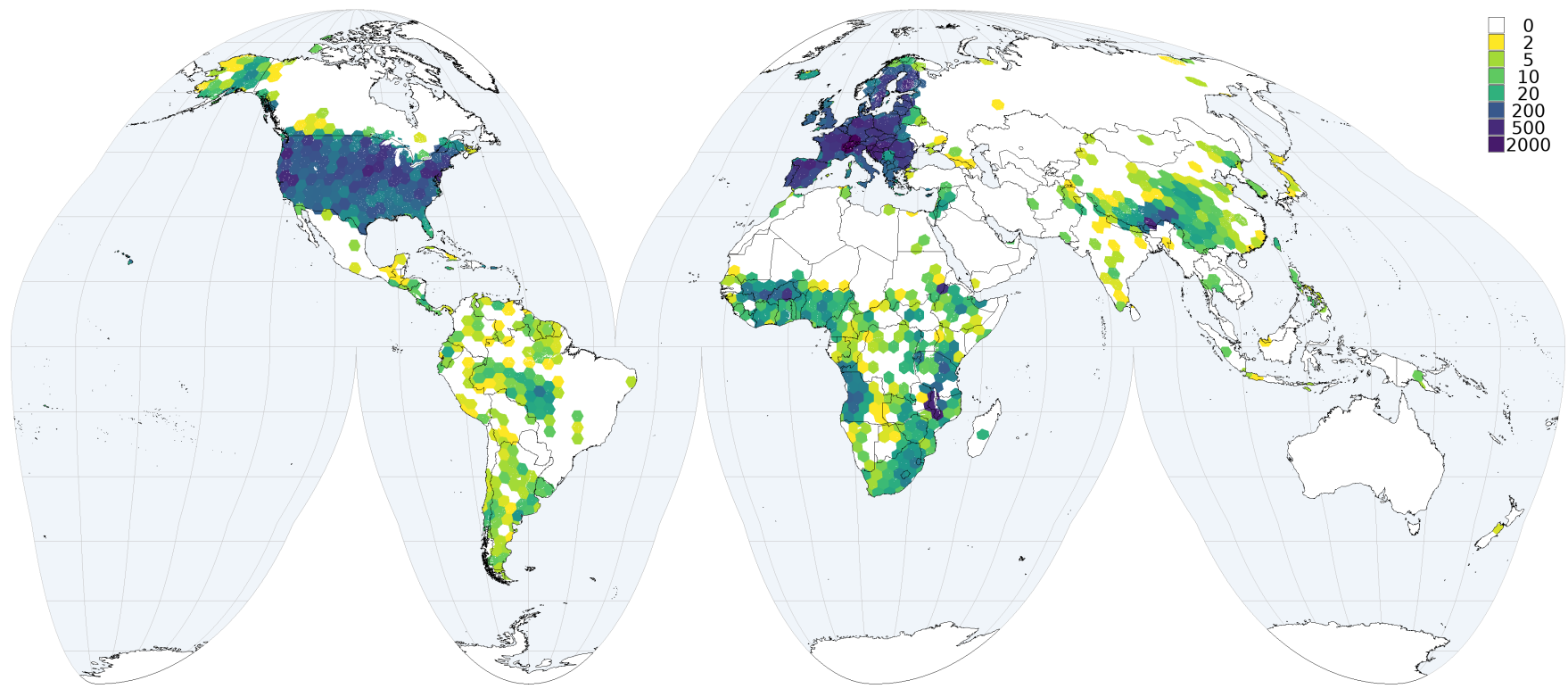

Figure S4. Number of observations for coarse fragments.

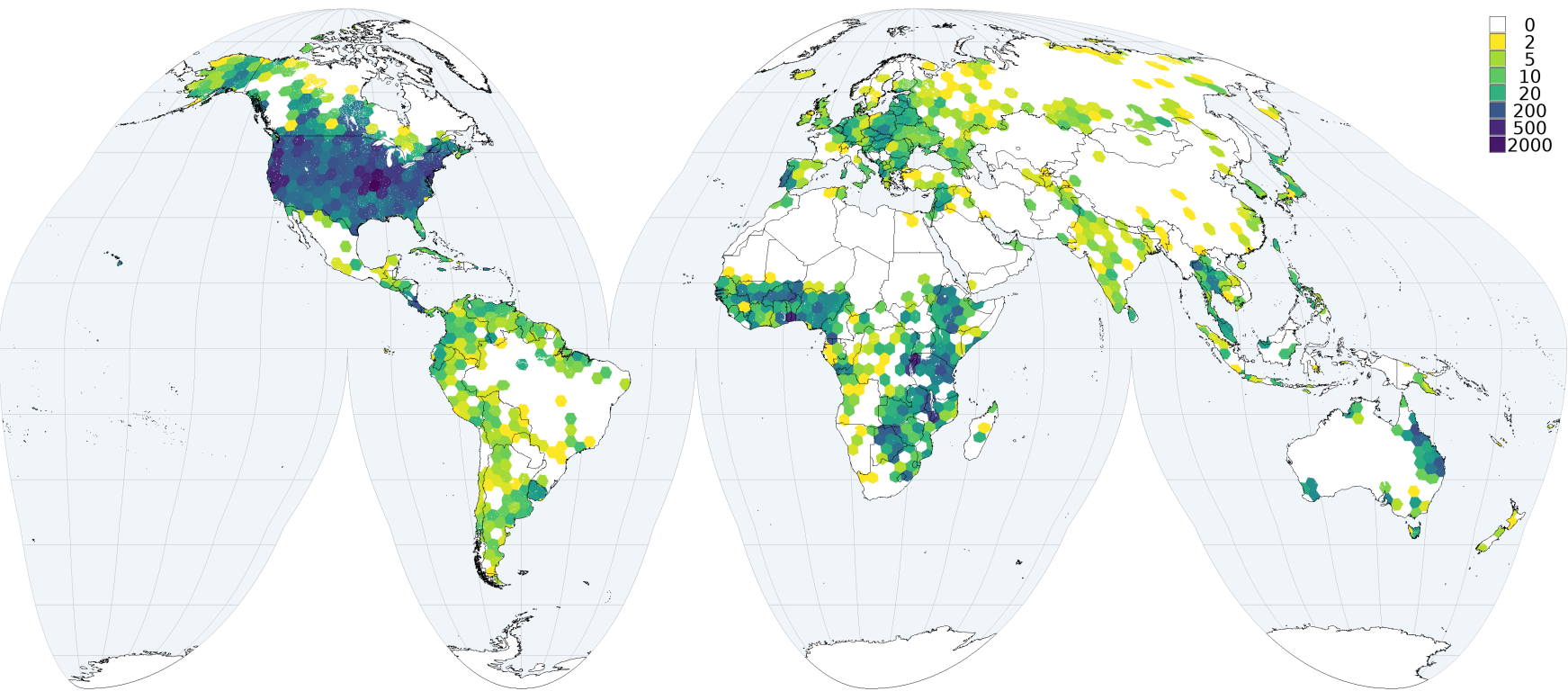

Figure S5. Number of observations for cation exchange capacity of the soil. 


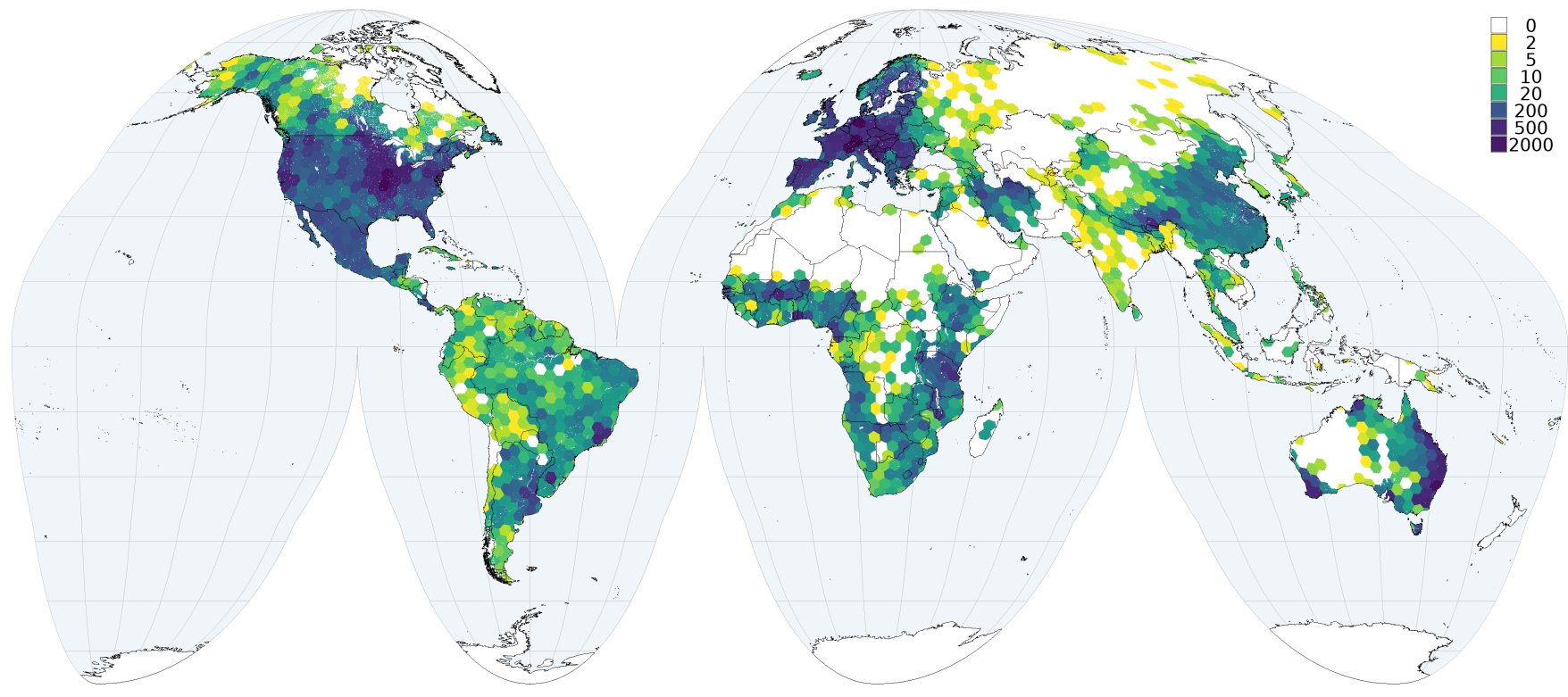

Figure S6. Number of observations for texture fractions.
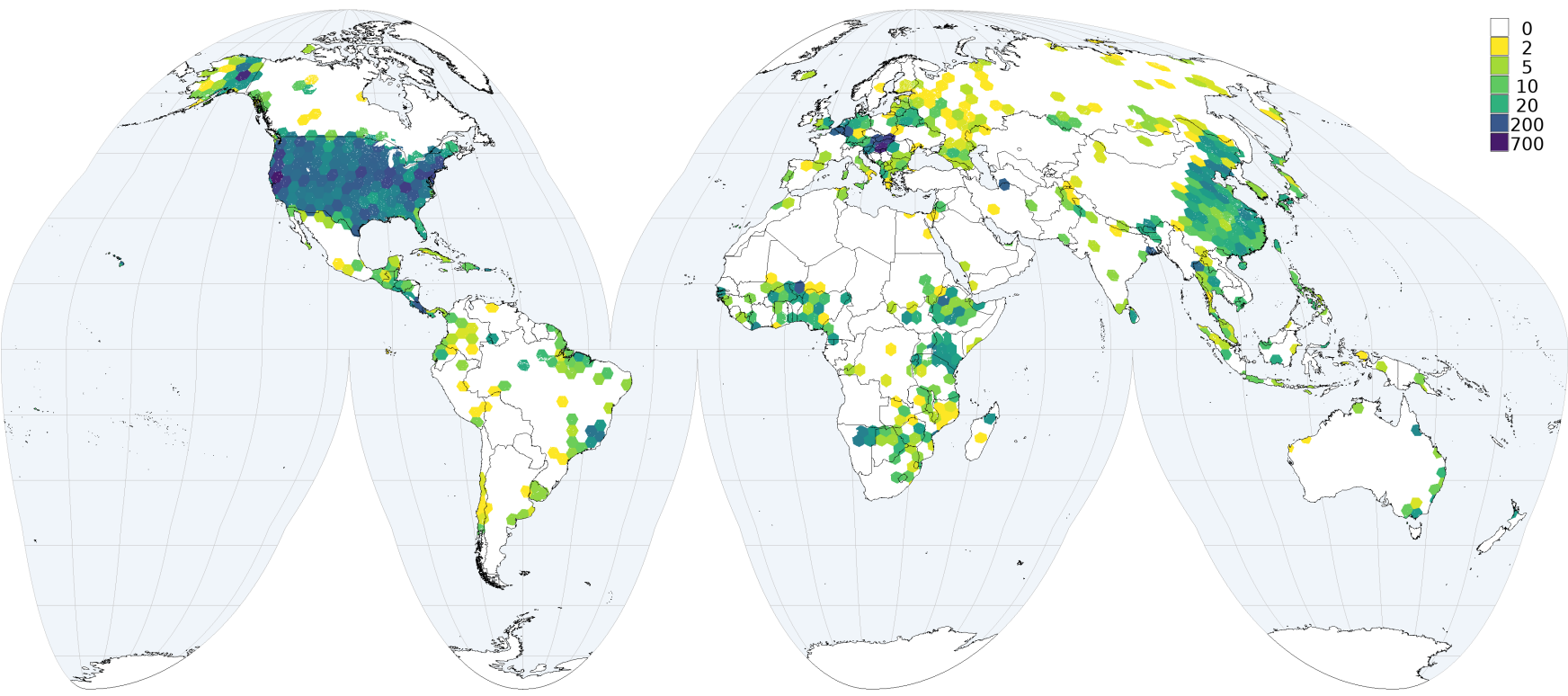

Figure S7. Number of observations for bulk density. 
2 Numerical evaluation (model performance metrics) by bio-climatic region and depth interval
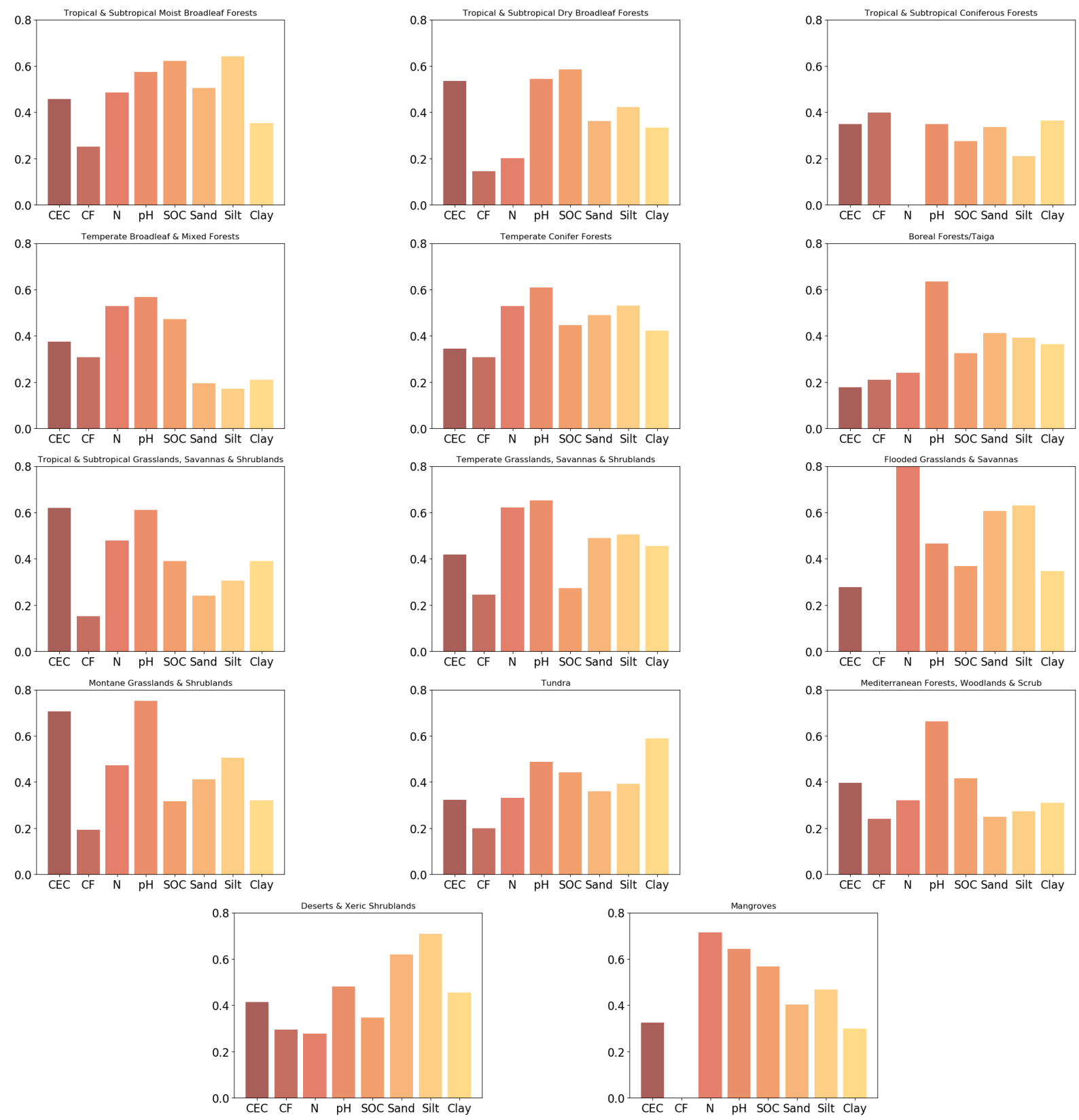

Figure S8. MEC for mean predictions by bio-climatic region. 

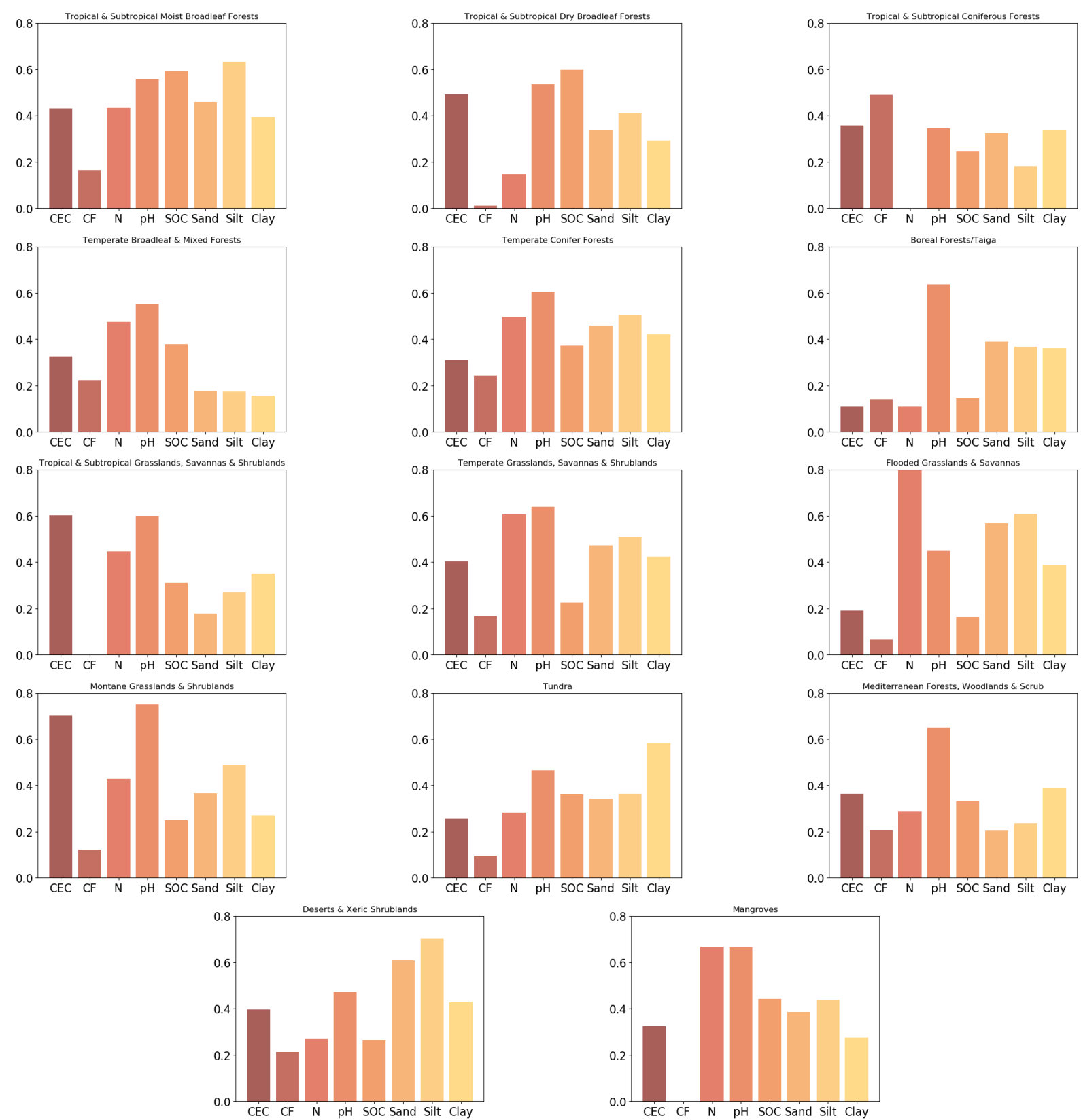

Figure S9. MEC for median predictions by bio-climatic region. 


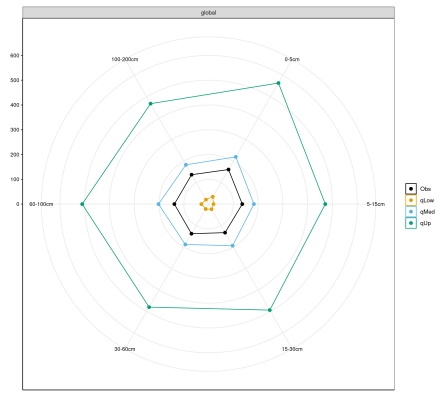

(a) Cation Exchange Capacity

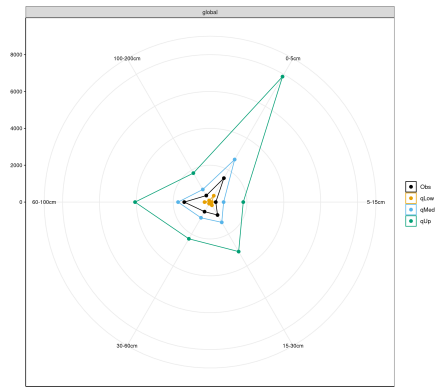

(c) Nitrogen

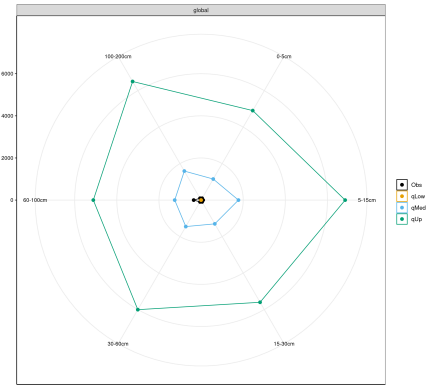

(b) Coarse Fragments

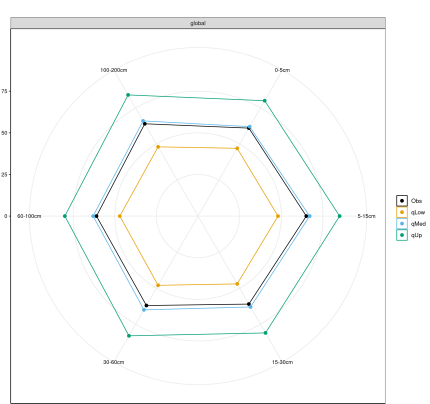

(d) Soil $\mathrm{pH}$

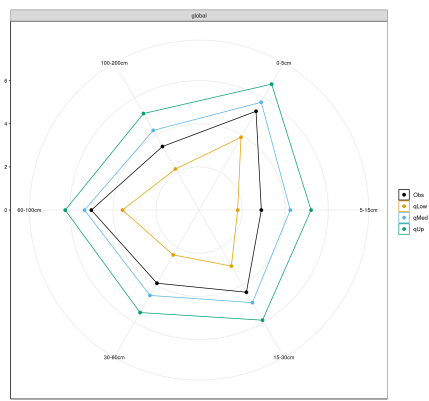

(e) Soil Organic Carbon

Figure S10. Summary statistics of the maps by standard depth interval compared with observed values for the corresponding interval. 